\title{
Psicoeducação do Transtorno do Déficit de Atenção/Hiperatividade: O Que, Como e Para Quem Informar?
}

Clarissa Tochetto de Oliveira*

Orcid.org/0000-0002-2624-8843

Ana Cristina Garcia Dias

Orcid.org/0000-0003-2312-3911

Universidade Federal do Rio Grande do Sul, Porto Alegre, RS, Brasil

\section{Resumo}

O objetivo deste estudo foi caracterizar as publicações científicas sobre psicoeducação do Transtorno do Déficit de Atenção/Hiperatividade (TDAH). Foi realizada uma revisão sistemática da literatura em oito bases de dados nacionais e internacionais. Utilizou-se a combinação das palavras-chave TDAH e psicoeducação. No total, foram encontrados 504 trabalhos completos, mas apenas 29 preencheram os critérios de inclusão para análise na íntegra. As informações foram organizadas nas categorias: conceito de psicoeducação, público-alvo, foco da psicoeducação do TDAH, variáveis relacionadas à psicoeducação do TDAH, e características das intervenções em psicoeducação do TDAH. Verificou-se que nem todas as publicações apresentam claramente o conceito de psicoeducação utilizado. Em geral, a psicoeducação tem sido destinada a familiares de pessoas com TDAH, e conduzida em formato de sessões grupais, palestras e manuais. A psicoeducação do TDAH contribui para maior conhecimento sobre o transtorno, adesão ao tratamento, qualidade de vida e menor intensidade dos sintomas do TDAH.

Palavras-chave: Transtorno da falta de atenção com hiperatividade, psicoeducação, revisão de literatura.

\section{Psychoeducation for Attention Deficit/Hyperactivity Disorder: What, How and Who Shall We Inform?}

\begin{abstract}
The aim of this study was to review scientific publications about Attention Deficit/Hyperactivity Disorder (ADHD) psychoeducation. A systematic review of literature was performed in eight national and international databases (Scielo Brazil, Index Psi, Pepsic, Lilacs, ScienceDirect, PsycINFO, Medline, and Scopus) using the combination of ADHD and psychoeducation as keywords. In total, 504 studies were found, although only 29 met the inclusion criteria. Afterwards, the information was organized into
\end{abstract}

Endereço para correspondência: Rua Ramiro Barcelos, 2600, Bairro Rio Branco, Porto Alegre, RS, Brasil 90035-003. Fone: (51) 3316-5066. E-mail: clarissa.tochetto@gmail.com

Agradecemos ao Conselho Nacional de Desenvolvimento Científico e Tecnológico (CNPq) e Fundação de Amparo à Pesquisa do Estado do Rio Grande do Sul (FAPERGS) pelo financiamento e à Karen Rech Braun pela colaboração como juíza. 
categories: the concept of psychoeducation, target population, focus of ADHD psychoeducation, variables related to ADHD psychoeducation, and the characteristics of intervention on ADHD psychoeducation. Results show that not all studies present the concept of psychoeducation adopted by the authors. In general, psychoeducation has focused on the relatives of people with ADHD and carried out as group sessions, lectures, and manuals. ADHD psychoeducation contributes to a better understanding of the disorder, treatment adherence, quality of life, and decreased symptoms of ADHD.

Keywords: Attention deficit disorder with hyperactivity, psychoeducation, literature review.

\section{La Psicoeducación del Trastorno por Déficit de Atención/Hiperactividad: Qué, Cómo ya Quién Informar?}

\section{Resumen}

El objetivo de este estudio fue caracterizar las publicaciones científicas sobre la psicoeducación del Trastorno por Déficit de Atención/Hiperactividad (TDAH). Se realizó una revisión sistemática de la literatura en ocho bases de datos nacionales e internacionales con la combinación de palabras clave psicoeducación y TDAH. En total, fueran encontrados 504 estudios, pero sólo 29 cumplieron los criterios de inclusión para el análisis en su totalidad. La información se organiza en categorías: concepto de la psicoeducación, la audiencia, el enfoque de la psicoeducación del TDAH, las variables relacionadas con la psicoeducación del TDAH, y características de las intervenciones en la psicoeducación del TDAH. Se ha encontrado que no todas las publicaciones muestran claramente el concepto de psicoeducación utilizado. En general, la psicoeducación se ha diseñado para familiares de personas con TDAH, y llevado a cabo en el formato de sesiones de grupo, conferencias y manuales. La psicoeducación del TDAH contribuye a un mayor conocimiento sobre el trastorno, la adherencia al tratamiento, calidad de vida y menor intensidad de los síntomas del TDAH.

Palabras clave: Trastorno por Déficit de Atención/Hiperactividad, psicoeducación, revisión de literatura.

O Transtorno do Déficit de Atenção/Hiperatividade (TDAH) é caracterizado por um padrão persistente de desatenção, hiperatividade e impulsividade (American Psychiatric Association [APA], 2013). Esses sintomas têm origem neurobiológica, e são decorrentes da incapacidade de inibir reações impulsivas e de considerar o futuro para guiar o comportamento (Barkley, Fischer, Smallish, \& Fletcher, 2002). Como consequência, pessoas diagnosticadas com esse transtorno tendem a apresentar prejuízos no âmbito acadêmico, profissional, familiar e social. $\mathrm{O}$ TDAH é crônico. Os sintomas iniciam antes dos 12 anos (APA, 2013) e tendem a permanecer ao longo da vida em aproximadamente $70 \%$ dos casos (Lara et al., 2009). Os sintomas modificam-se conforme o período do desenvolvimento no qual o indivíduo se encontra. Na idade adulta, a desatenção pode se manifestar pela evitação de atividades que exijam manutenção da atenção, como assistir a filmes e ler; a hiperatividade, na forma de inquietação interna ou desconforto; e a impulsividade pode aparecer em decisões sem pensar nas consequências (Conners, 2009).

Independente da fase do desenvolvimento, o tratamento para pessoas que apresentam esse transtorno envolve a combinação de medicação (para minimizar os sintomas de desatenção, hiperatividade e impulsividade) e a adoção de intervenções psicológicas, a exemplo das propostas pela terapia cognitivo-comportamental (TCC), para desenvolver estratégias para lidar com os sintomas residuais (Mongia \& Hechtman, 2016; Sprich, Safren, Finkelstein, Remmert, \& Hammerness, 2016). Contudo, a não adesão ao tratamento pode variar de $13,2 \%$ a $64,0 \%$ (McCarthy, 2014) por fatores associados à falta de conhecimento sobre o TDAH, às características 
da medicação e aos objetivos propostos no tratamento (Charach \& Fernandez, 2013). Portanto, o fornecimento de informações sobre esses aspectos para os pacientes e seus familiares pode ser fundamental para a busca e a manutenção do tratamento do TDAH.

O termo psicoeducação começou a ser utilizado na década de 80 , referindo-se à transmissão de informações sobre os transtornos mentais para familiares e pacientes psicóticos. Na década de 90, a psicoeducação passou a ser desenvolvida junto a outros grupos que apresentavam outros transtornos mentais (como transtorno do humor bipolar, transtorno do estresse pós-trauma etc.; Bonsack, Rexhaj, \& Favrod, 2015). Atualmente, esse termo se refere tanto à oferta de informações relevantes aos pacientes sobre o transtorno (diagnóstico, etiologia, funcionamento), o tratamento e o prognóstico, quanto à busca por esclarecimento de dúvidas e correções de informações distorcidas. A psicoeducação tem por objetivo ampliar o conhecimento do paciente/ familiar sobre seu problema (Menezes \& Souza, 2012; Swadi, Bobier, Price, \& Craig, 2010) a fim de aumentar a compreensão da sua condição, auxiliar na tomada de decisões com base em informações confiáveis e promover maior adesão ao tratamento (Bégin, Bluteau, Arseneault, \& Pronovost, 2012; Bonsack et al., 2015; Swaminath, 2009). Ela deve ocorrer de forma didática e em linguagem adequada ao público-alvo, que pode ser constituído por pacientes, familiares, educadores e profissionais da saúde. A transmissão desses conhecimentos assume diferentes formatos: individual ou grupal, palestras, rodas de conversas, manuais, vídeos ou biblioterapia (Bai, Wang, Yang, \& Niu, 2015). Dentre os diversos benefícios da psicoeducação, destacam-se o aumento do conhecimento sobre o transtorno, da motivação para a mudança, da participação no tratamento, da satisfação com o tratamento psicológico, a maior adesão ao mesmo e a redução de recaídas (Burlingame, Ridge, Matasuno, Hwang, \& Ernshaw, 2006; Knapp, 2004; Nussey, Pistrang, \& Murphy, 2013).

Programas de psicoeducação são mais efetivos na redução dos sintomas do TDAH quando comparados ao tratamento medicamentoso iso- lado, o que indica a necessidade de considerar a psicoeducação como uma abordagem adicional válida para o tratamento do TDAH (Ferrin et al., 2016). Ademais, é possível que a identificação de informações relevantes para cada caso, como adequar a transmissão dessas informações de acordo com as características do público-alvo, e como verificar se a mensagem foi, de fato, compreendida contribuam para o engajamento do paciente no tratamento (Burlingame et al., 2006; Knapp, 2004). Por isso, o objetivo deste estudo foi caracterizar as publicações científicas sobre psicoeducação do TDAH. Em especial, buscou-se identificar o conceito de psicoeducação utilizado pelos pesquisadores, a quem a psicoeducação se destina, o foco da psicoeducação, variáveis relacionadas e intervenções em psicoeducação para o TDAH.

\section{Método}

Realizou-se uma revisão sistemática da literatura sobre psicoeducação do TDAH. A busca foi realizada nas bases de dados Scielo Brasil, Index Psi, Pepsic, Lilacs, ScienceDirect, PsycINFO, Medline, Scopus. Optou-se por essas bases de dados por oferecerem acesso ao texto completo de publicações nacionais (Scielo Brasil, Index Psi e Pepsic) e internacionais (Lilacs, ScienceDirect, PsycINFO, Medline e Scopus) no meio eletrônico. Foi utilizada a combinação entre os descritores TDAH/ADHD ou Transtorno do Déficit de Atenção/Hiperatividade/Attention Deficit/Hyperactivity Disorder de um lado e, de outro, psicoeducação/psychoeducation, biblioterapia/bibliotherapy ou intervenção psicoeducativa/psychoeducative interventions, no campo resumo/abstract. Essas palavras-chave foram escolhidas com base no objetivo do estudo.

No total, foram encontrados 504 trabalhos completos. As buscas nas bases de dados brasileiras identificaram apenas três trabalhos utilizando os descritores combinados. Destes, um foi incorporado na amostra final (Mesquita, Porto, Rangé, \& Ventura, 2009), os outros dois estavam duplicados ou indisponíveis. Os 501 artigos restantes foram recuperados de bases internacionais. Os resumos das 504 publicações 
foram lidos por dois juízes a fim de delimitar a amostra de trabalhos a serem analisados na íntegra. Os critérios de inclusão dos artigos para participação na amostra final deste estudo foram: (a) abordar psicoeducação do TDAH, (b) ser redigido em Português, Inglês ou Espanhol, e (c) artigos originais publicados em periódicos científicos, ou seja, não foram incluídos livros, capí- tulos de livros, dissertações e teses. Nesta etapa de análise, foram excluídos 475 (Figura 1). O índice de concordância entre juízes foi de 89,65. As discordâncias foram solucionadas pelo acesso às publicações na sua totalidade e reavaliação das mesmas de acordo com o primeiro critério de inclusão.

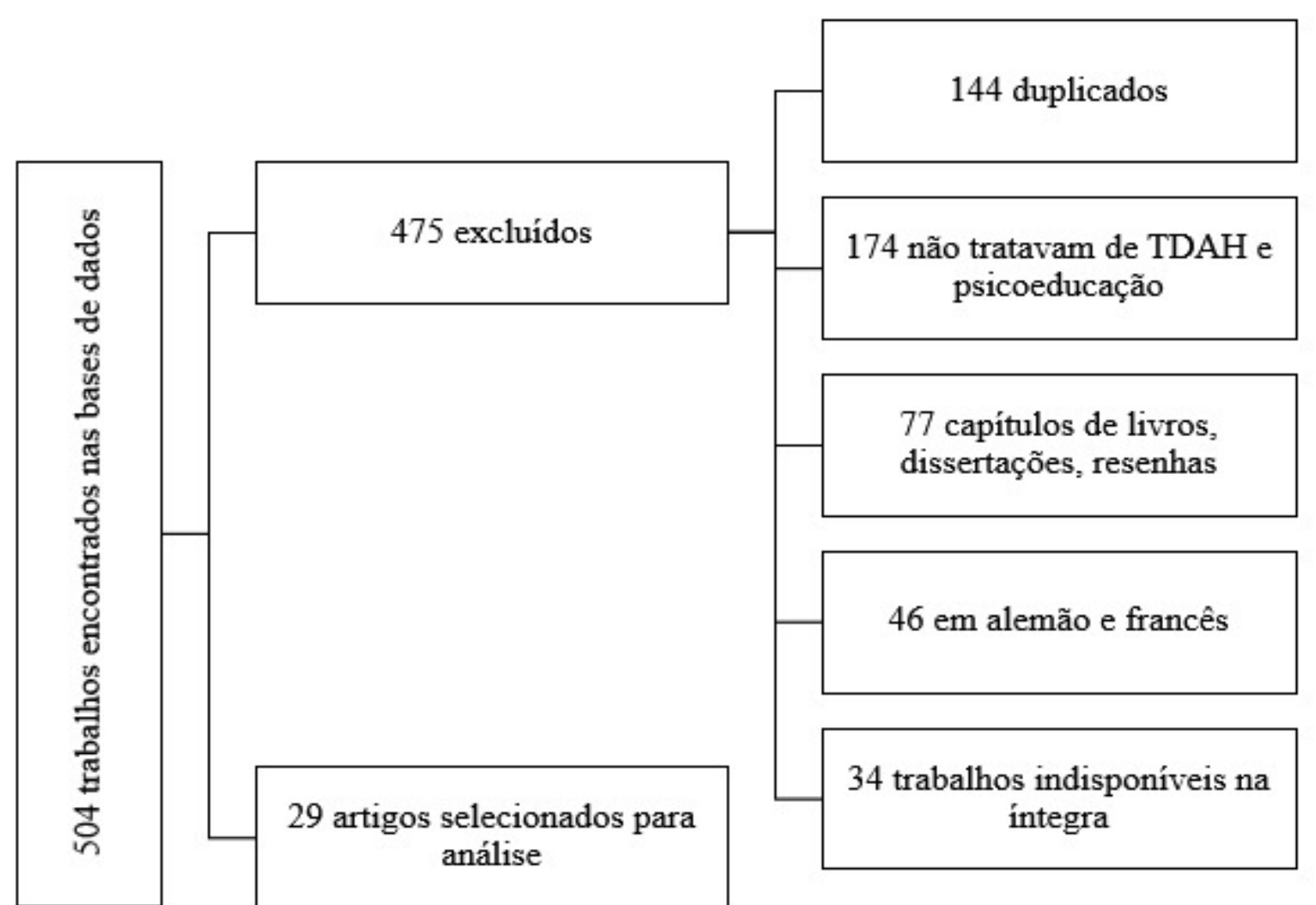

Figura 1. Procedimento de triagem dos artigos recuperados

A amostra final foi constituída por 29 publicações internacionais, que foram avaliadas em quatro etapas: leitura exploratória, seletiva, analítica e interpretativa (Gil, 2006). Na primei$\mathrm{ra}$, os textos foram lidos na sua totalidade. $\mathrm{Na}$ segunda, realizou-se uma leitura aprofundada do método, resultados e conclusões. Na terceira, as informações foram ordenadas de forma a identificar as principais características da psicoeducação (conceito, público-alvo, foco e variáveis relacionadas). Por fim, na última etapa, as informações foram agrupadas em categorias definidas a priori, com base no objetivo deste estudo:

1. Conceito de psicoeducação,

2. Público-alvo,
3. Foco da psicoeducação do TDAH,

4. Variáveis relacionadas à psicoeducação do TDAH,

5. Características das intervenções em psicoeducação do TDAH.

\section{Resultados e Discussão}

Foram analisados 29 estudos internacionais. Destes, 14 apresentavam delineamento experimental (Aguiar et al., 2014; Anderson \& Guthery, 2015; Bai et al., 2015; Ferrin et al., 2014; Hirvikoski, Waaler, Lindström, Bölte, \& Jokinen, 2015; Janssen et al., 2014; Kądziela-Olech, 2012; Korzeniowsk \& Ison, 2008; Long, Rick- 
ert, \& Ashcraft, 1993; McCarty, Vander Stoep, Violette, \& Myers, 2015; McCleary \& Ridley, 1999; Montoya et al., 2014; Myers, Vander Stoep, Thompson, Zhou, \& Unützer, 2010; Vidal et al., 2013), oito eram revisões de literatura (Asherson, 2012; Hernández \& Gutiérrez 2014; Hill, 2015; Knouse, Cooper-Vince, Sprich, \& Safren, 2008; Montoya, Colom, \& Ferrin, 2011; Murphy, 2005; Nussey et al., 2013; Young, 1999), quatro eram estudo de levantamento (Altin, Altin, \& Semerci, 2016; Bussing et al., 2012; Coletti et al., 2012; Palacios-Cruz et al., 2013) e três, estudos de caso (Hogue, Bobek, \& Evans, 2014; Levine \& Anshel, 2011; Mesquita et al.,
2009). De modo geral, os estudos com delineamento experimental e estudos de caso consistem em intervenções de psicoeducação do TDAH destinadas a familiares de crianças/adolescentes com TDAH. As revisões de literatura referem-se à psicoeducação do TDAH para crianças, adolescentes, adultos, familiares, escola e profissionais na saúde. Por fim, os estudos de levantamento buscaram avaliar o conhecimento de adolescentes, genitores e professores sobre o TDAH. Informações sobre o conceito do psicoeducação adotado pelos pesquisadores, público-alvo, foco da psicoeducação e variáveis relacionadas estão descritas na Tabela 1.

Tabela 1

Características da Psicoeducação do TDAH nos Estudos Analisados

\begin{tabular}{|c|c|c|c|c|}
\hline $\begin{array}{l}\text { Autores } \\
\text { (ano) }\end{array}$ & Público-alvo & Conceito de psicoeducação & Foco da psicoeducação & $\begin{array}{l}\text { Variáveis } \\
\text { relacionadas }\end{array}$ \\
\hline $\begin{array}{l}\text { Aguiar et al. } \\
(2014)\end{array}$ & $\begin{array}{l}\text { Professores } \\
\text { do Ensino } \\
\text { Fundamental }\end{array}$ & Não há & $\begin{array}{l}\text { TDAH (sintomas, etiologia, } \\
\text { vinhetas clínicas, estratégias } \\
\text { para lidar com } \\
\text { os transtornos) }\end{array}$ & \\
\hline $\begin{array}{l}\text { Altin et al. } \\
(2016)\end{array}$ & $\begin{array}{l}\text { Adultos } \\
\text { com } \\
\text { TDAH }\end{array}$ & $\begin{array}{l}\text { Parte do tratamento que ajuda } \\
\text { pacientes e familiares a dar sentido } \\
\text { aos sintomas, transtorno } \\
\text { e seus prejuízos. }\end{array}$ & Não há & Não há \\
\hline $\begin{array}{l}\text { Anderson } \\
\& \text { Guthery } \\
(2015)\end{array}$ & $\begin{array}{l}\text { Pais de } \\
\text { crianças com } \\
\text { TDAH ou } \\
\text { traços do } \\
\text { TDAH }\end{array}$ & Não há & Mindfulness & Estresse \\
\hline $\begin{array}{l}\text { Asherson } \\
(2012)\end{array}$ & $\begin{array}{l}\text { Adolescentes } \\
\text { e adultos } \\
\text { com TDAH }\end{array}$ & Não há & $\begin{array}{c}\text { Medicação } \\
\text { e estratégias } \\
\text { de enfrentamento }\end{array}$ & Não há \\
\hline $\begin{array}{l}\text { Bai et al. } \\
(2015)\end{array}$ & $\begin{array}{l}\text { Famílias } \\
\text { de crianças } \\
\text { (6-16 anos) } \\
\text { com TDAH }\end{array}$ & $\begin{array}{c}\text { Programa terapêutico focado } \\
\text { na comunicação didática } \\
\text { de informações e estratégias } \\
\text { de enfrentamento para pacientes, } \\
\text { familiares ou escola. }\end{array}$ & $\begin{array}{l}\text { TDAH (sintomas, causas, } \\
\text { prejuízos ao longo } \\
\text { da vida), medicação } \\
\text { (efeitos colaterais, } \\
\text { eficácia e segurança), } \\
\text { opções de tratamento } \\
\text { (eficácia). }\end{array}$ & $\begin{array}{l}\text { Conhecimento } \\
\text { sobre TDAH } \\
\text { e medicação }\end{array}$ \\
\hline $\begin{array}{l}\text { Bussing et al. } \\
(2012)\end{array}$ & $\begin{array}{l}\text { Adolescentes } \\
\text { com alto e } \\
\text { baixo risco } \\
\text { de apresentar } \\
\text { TDAH }\end{array}$ & $\begin{array}{c}\text { Health Belief Model (HBM) } \\
\text { avalia percepções do paciente que } \\
\text { podem ser foco de psicoeducação, } \\
\text { como severidade do transtorno } \\
\text { e benefício das intervenções. } \\
\text { Psicoeducação deve ser culturalmente } \\
\text { sensível para facilitar a troca de } \\
\text { informações entre profissionais, }\end{array}$ & Não há & $\begin{array}{l}\text { Informa } \\
\text { conhecimento } \\
\text { de adolescentes } \\
\text { e genitores sobre } \\
\text { o TDAH, bem } \\
\text { como as fontes } \\
\text { de informação } \\
\text { preferidas. }\end{array}$ \\
\hline
\end{tabular}




\begin{tabular}{|c|c|c|}
\hline $\begin{array}{l}\text { Autores } \\
\text { (ano) }\end{array}$ & Público-alvo & Conceito de psicoeducação \\
\hline $\begin{array}{l}\text { Coletti et al. } \\
(2012)\end{array}$ & $\begin{array}{c}\text { Pais de } \\
\text { crianças com } \\
\text { TDAH }\end{array}$ & Não há \\
\hline $\begin{array}{l}\text { Ferrin et al. } \\
(2014)\end{array}$ & $\begin{array}{l}\text { Famílias de } \\
\text { crianças/ } \\
\text { adolescentes } \\
\text { com TDAH }\end{array}$ & $\begin{array}{l}\text { Abordagem sistemática e didática, } \\
\text { adequada para informar pacientes } \\
\text { e familiares sobre o transtorno } \\
\text { e tratamento, facilitando a } \\
\text { compreensão e a forma de } \\
\text { enfrentamento do transtorno. }\end{array}$ \\
\hline $\begin{array}{l}\text { Hernández } \\
\& \text { Gutiérrez } \\
(2014)\end{array}$ & $\begin{array}{l}\text { Pacientes, } \\
\text { familiares e } \\
\text { educadores }\end{array}$ & $\begin{array}{c}\text { Estratégia para transmitir } \\
\text { informações sobre o transtorno } \\
\text { a pacientes, pais e educadores } \\
\text { baseados na utilidade } \\
\text { e veracidade científica. }\end{array}$ \\
\hline Hill (2015) & $\begin{array}{l}\text { Crianças, } \\
\text { pais, } \\
\text { escolas e } \\
\text { profissionais } \\
\text { de saúde } \\
\text { básica }\end{array}$ & Não há \\
\hline $\begin{array}{l}\text { Hirvikoski } \\
\text { et al. (2015) }\end{array}$ & $\begin{array}{c}\text { Adultos } \\
\text { com TDAH } \\
\text { e outros } \\
\text { significativos }\end{array}$ & $\begin{array}{l}\text { Intervenção baseada em } \\
\text { evidências para diversos } \\
\text { transtornos psiquiátricos para } \\
\text { empoderar pacientes e familiares } \\
\text { com conhecimento sobre } \\
\text { o transtorno e seu tratamento }\end{array}$ \\
\hline
\end{tabular}

Hogue et al. Adolescentes (2014) com TDAH e familiares

Janssen et al. (2014)

Pais de
crianças e
adolescentes

com TDAH

$\begin{array}{lc}\begin{array}{l}\text { Kądziela- } \\ \text { Olech (2012) }\end{array} & \begin{array}{c}\text { Pais de } \\ \text { crianças com } \\ \text { TDAH }\end{array} \\ \begin{array}{l}\text { Knouse et al. } \\ (2008)\end{array} & \begin{array}{c}\text { Adultos com } \\ \text { TDAH }\end{array} \\ \begin{array}{l}\text { Korzeniowsk } \\ \text { \& Ison (2008) }\end{array} & \begin{array}{c}\text { Pais e } \\ \text { professores } \\ \text { de crianças } \\ \text { com TDAH }\end{array}\end{array}$

Intervenções educacionais interativas que fornecem informações estruturadas sobre o TDAH. Essas informações são oferecidas em formato de fácil compreensão e possibilita o desenvolvimento de um perfil único dos sintomas do TDAH relacionado às características de cada paciente.

Não há
Informação sobre o transtorno e tratamento para pacientes, familiares e professores.

Não há

Não há
Foco da psicoeducação

TDAH e medicação.

TDAH e estratégias

comportamentais para manejo dos sintomas e de comportamento desafiante.

Corrigir crenças distorcidas sobre etiologia, sintomas, diagnóstico, tratamento e estratégias de manejo dos sintomas.

TDAH (causas, comorbidades, prejuízos), medicação (limitações)

TDAH na vida adulta, prejuízos nos relacionamentos e no trabalho, tratamento farmacológico e psicológico, estratégias de enfrentamento e serviços disponíveis.

Sintomas, curso do TDAH, funções executivas, efeitos em diversos domínios de funcionamento (família, escola, pares), e diferenças individuais associadas ao TDAH em adolescentes.

Não há informações sobre a psicoeducação para pais

TDAH (etiologia, diagnóstico) e tratamento.

TDAH

TDAH, dificuldades escolares, estratégias para lidar com os sintomas.
Variáveis

relacionadas

Decisão de aderir ao uso da medicação

Sintomas do TDAH, psicopatologia, qualidade de vida, estresse familiar.

Não há

Não há

Satisfação com intervenção, taxa de abandono, autoestima, conhecimento sobre TDAH

(V ou F), qualidade de vida.

Desempenho escolar, autoeficácia, autorregulação de habilidades acadêmicas, colaboração entre pais e escola.

Sintomas do TDAH, saúde mental, qualidade de vida, adesão ao tratamento.

Severidade dos sintomas do TDAH

Não há

Sintomas do TDAH, comportamentos problemáticos, estratégias educativas. 
e Para Quem Informar?

\begin{tabular}{lcc}
$\begin{array}{l}\text { Autores } \\
\text { (ano) }\end{array}$ & Público-alvo & Conceito de psicoeducação \\
\hline $\begin{array}{l}\text { Levine \& } \\
\text { Anshel }\end{array}$ & $\begin{array}{l}\text { Criança (oito } \\
\text { anos), mãe e } \\
\text { professora }\end{array}$ & $\begin{array}{c}\text { Oferecer informações sobre o } \\
\text { para resolico, desenvão de problemas de } \\
\text { maneira independente }\end{array}$
\end{tabular}

Long et al. Famílias com Biblioterapia: leitura utilizada como (1993) crianças (6-
11 anos) com

TDAH

$\begin{array}{lrr}\begin{array}{l}\text { McCarty } \\ \text { et al. (2015) }\end{array} & \begin{array}{c}\text { Cuidadores } \\ \text { de crianças } \\ \text { com TDAH }\end{array} & \begin{array}{c}\text { Imagens, gráficos, e textos breves } \\ \text { para auxiliar cuidadores e crianças } \\ \text { a compreender que o TDAH e } \\ \text { comportamentos decorrentes são } \\ \text { neurológicos, bem como os objetivo } \\ \text { do tratamento. }\end{array}\end{array}$

McCleary \& Pais de Ridley (1999) adolescentes com TDAH

$\begin{array}{lc}\begin{array}{l}\text { Mesquita } \\ \text { et al. (2009) }\end{array} & \begin{array}{c}\text { Adulto com } \\ \text { TDAH }\end{array} \\ \text { Montoya } & \begin{array}{c}\text { Pais e } \\ \text { et al. (2011) } \\ \text { professores } \\ \text { de crianças e } \\ \text { adolescentes } \\ \text { com TDAH }\end{array}\end{array}$

Montoya et al. Pais de (2014) crianças com

TDAH
Busca melhorar as habilidades para lidar com os problemas associados ao TDAH por meio o fornecimento de informações sobre o transtorno, aumento das habilidades parentais (negociação e manejo de conflitos) e apoio ao seu senso de competência.

Permite que os pacientes reconheçam seus sintomas, danos e criem estratégias para o manejo destes

Novo paradigma de tratamento que inclui informações sobre o transtorno e tratamento, empodera o paciente $\mathrm{e}$ é considerada uma prática baseada em evidências para transtornos psiquiátricos na adultez.

Não há
Foco da psicoeducação

Variáveis

relacionadas

\author{
Sintomas \\ depressivos \\ (tristeza e baixa \\ autoestima)
} pares, engajar a criança na participação das intervenções

Uso de princípios da aprendizagem social no manejo comportamental das crianças

TDAH (etiologia, neurobiologia e neuroquímica, comorbidades, tratamentos, impacto nas fases da vida), estratégias de reforço e extinção de comportamentos, e habilidades parentais para lidar sintomas

TDAH e problemas associados, estratégias de enfrentamento.

TDAH, modelo cognitivo, tratamento farmacológico e comorbidades

TDAH, comorbidades, adesão ao tratamento, relação parental, entre pares e com professores, habilidades sociais, qualidade de vida.

Programa psicoeducativo sobre TDAH (etiologia, sintomas, comorbidades, diagnóstico, tratamento, prognóstico), estratégias de enfrentamento e suporte, habilidades sociais e parentais, e resolução de problemas de atenção.

\section{Sintomas} do TDAH, comportamentos da criança, conhecimento sobre técnicas comportamentais

Engajamento dos cuidadores.

Não há

\section{Depressão}

Não há

\author{
Tratamento
} medicamentoso 


\begin{tabular}{|c|c|c|c|c|}
\hline $\begin{array}{l}\text { Autores } \\
\text { (ano) }\end{array}$ & Público-alvo & Conceito de psicoeducação & Foco da psicoeducação & $\begin{array}{l}\text { Variáveis } \\
\text { relacionadas }\end{array}$ \\
\hline $\begin{array}{l}\text { Murphy } \\
(2005)\end{array}$ & $\begin{array}{l}\text { Adolescentes } \\
\text { e adultos } \\
\text { com TDAH }\end{array}$ & $\begin{array}{l}\text { O conhecimento pode auxiliar } \\
\text { pacientes e familiares a lidar com } \\
\text { o TDAH de forma mais efetiva, } \\
\text { ajudar pacientes a identificar o que } \\
\text { os prejudica, e a estabelecer metas } \\
\text { de tratamento realistas. Explicar } \\
\text { o diagnóstico do TDAH e como o } \\
\text { paciente preenche os critérios do } \\
\text { DSM pode ajudar a desmistificar o } \\
\text { diagnóstico e contextualizar o TDAH } \\
\text { na experiência de cada paciente. }\end{array}$ & $\begin{array}{l}\text { TDAH (critérios } \\
\text { diagnósticos, impacto } \\
\text { na vida do paciente, } \\
\text { tratamentos) }\end{array}$ & Não há \\
\hline $\begin{array}{l}\text { Myers et al. } \\
(2010)\end{array}$ & $\begin{array}{l}\text { Crianças } \\
\text { (6-12 anos) }\end{array}$ & Não há & $\begin{array}{l}\text { TDAH } \\
\text { (etiologia e manejo) }\end{array}$ & $\begin{array}{l}\text { Sintomas do } \\
\text { TDAH, adesão } \\
\text { e satisfação com } \\
\text { o tratamento }\end{array}$ \\
\hline $\begin{array}{l}\text { Nussey et al. } \\
(2013)\end{array}$ & $\begin{array}{l}\text { Pais, } \\
\text { professores } \\
\text { e pares } \\
\text { (criança } \\
\text { e adulto) }\end{array}$ & Não há & TDAH e Tourette & Não há \\
\hline $\begin{array}{l}\text { Palacios-Cruz } \\
\text { et al. }(2013)\end{array}$ & Professores & Não há & $\begin{array}{c}\text { Avaliação dos } \\
\text { conhecimentos } \\
\text { de professores sobre } \\
\text { o assunto }\end{array}$ & $\begin{array}{l}\text { Conhecimentos } \\
\text { e crenças sobre } \\
\text { TDAH }\end{array}$ \\
\hline $\begin{array}{l}\text { Vidal et al. } \\
\text { (2013) }\end{array}$ & $\begin{array}{l}\text { Adultos } \\
\text { com } \\
\text { TDAH }\end{array}$ & $\begin{array}{l}\text { Intervenção focada na compreensão } \\
\text { do transtorno para melhorar o } \\
\text { entendimento e conscientização dos } \\
\text { pacientes sobre a doença. }\end{array}$ & $\begin{array}{l}\text { TDAH (sintomas, } \\
\text { causas, prejuízos) e } \\
\text { tratamento (medicamento), } \\
\text { relaxamento e modelo } \\
\text { cognitivo do TDAH }\end{array}$ & $\begin{array}{c}\text { Sintomas do } \\
\text { TDAH, } \\
\text { depressão, } \\
\text { qualidade de vida }\end{array}$ \\
\hline $\begin{array}{l}\text { Young } \\
(1999)\end{array}$ & $\begin{array}{l}\text { Adultos } \\
\text { com } \\
\text { TDAH }\end{array}$ & $\begin{array}{l}\text { Corrige concepções sobre o transtorno } \\
\text { e fornece informações sobre o TDAH. }\end{array}$ & $\begin{array}{l}\text { TDAH (etiologia, } \\
\text { prognóstico e fatores } \\
\text { associados) }\end{array}$ & $\begin{array}{l}\text { Autoestima, } \\
\text { adesão ao } \\
\text { tratamento }\end{array}$ \\
\hline
\end{tabular}

\section{Conceito de Psicoeducação}

A psicoeducação é resultado da integração de diversas teorias e modelos clínicos complementares, tais como teoria cognitivo-comportamental, teoria da aprendizagem, modelo de prática de grupos, modelo de estresse e enfrentamento, modelo do suporte social, entre outras (Lukens \& McFarlane, 2004). Por exemplo, a psicoeducação pode ser utilizada como intervenção cognitiva isolada (Bäuml, Froböse, Kraemer, Rentrop, \& Pitschel-Walz, 2006) ou como estratégia na TCC para aumentar a compreensão do paciente sobre o que este apresenta e engajá-lo no tratamento (Knapp, 2004). Por isso, a definição do que os pesquisadores entendem por psicoeducação é relevante para facilitar a compreensão de profissionais interessados em utilizar os resultados dos estudos.

Alguns artigos analisados não apresentaram o conceito de psicoeducação utilizado (Aguiar et al., 2014; Anderson \& Guthery, 2015; Asherson, 2012; Coletti et al., 2012; Hill, 2015; Janssen et al., 2014; Knouse et al., 2008; Korzeniowsk \& Ison, 2008; Montoya et al., 2014; Myers et al., 2010; Nussey et al., 2013; Palacios-Cruz et al., 2013). Um estudo (Murphy, 2005) apresenta os benefícios de pacientes e familiares terem conhecimento sobre o TDAH, como identificar o que os prejudica e como desenvolver metas de tratamento rea- 
listas, mas não uma definição clara de psicoeducação.

Outros estudos analisados definiram psicoeducação como sendo uma forma de intervenção interativa entre profissional e paciente/familiares (Bai et al., 2015; Hirvikoski et al., 2015; Hogue et al., 2014; Montoya et al., 2011). A intervenção psicoeducativa avalia percepções sobre o transtorno e o tratamento (Bussing et al., 2012; Young, 1999) para, então, comunicar informações sobre o TDAH e as estratégias de enfrentamento de forma sistemática e didática (Bai et al., 2015; Ferrin, et al., 2014; Hogue et al., 2014; Montoya et al., 2011). Tem o objetivo de auxiliar na compreensão do TDAH, das consequências do mesmo e dos objetivos do tratamento (Altin et al., 2016; Hernández \& Gutiérrez 2014; Kądziela-Olech, 2012; Levine \& Anshel, 2011; McCarty et al., 2015; Mesquita et al., 2009; Vidal et al., 2013; Young, 1999), bem como empoderar pacientes e familiares (Hirvikoski et al., 2015) para melhorar suas habilidades para lidar com os problemas associados ao transtorno (Levine \& Anshel, 2011; McCleary \& Ridley, 1999; Mesquita et al., 2009). Pode-se fazer uso de biblioterapia, que consiste na leitura como técnica terapêtica, uma vez que manuais de autoajuda podem ser efetivos para uma variedade de problemas (Long et al., 1993).

Esses resultados mostram a necessidade de definir claramente o que os autores entendem por psicoeducação e como a mesma está sendo aplicada: como intervenção principal ou estratégia de tratamento mais abrangente. A especificação da forma como a mesma tem sido aplicada no contexto clínico e comunitário para diversos públicos-alvo também pode ser útil para clínicos e pesquisadores (Lukens \& McFarlane, 2004).

\section{Foco da Psicoeducação do TDAH}

O objetivo da psicoeducação é transmitir informações relevantes ao paciente ou interessados sobre determinado assunto (Menezes \& Souza, 2012; Swadi et al., 2010). Na amostra analisada, a psicoeducação do TDAH abordou o próprio transtorno e as opções de tratamento. Em especial, a psicoeducação do TDAH em si especificou os seguintes aspectos: (a) sintomas do transtorno (Aguiar et al., 2014; Bai et al., 2015; Coletti et al., 2012; Hernández \& Gutiérrez 2014; Hirvikoski et al., 2015; Hogue et al., 2014; Kądziela-Olech, 2012; Levine \& Anshel, 2011; Mesquita et al., 2009; Montoya et al., 2014; Murphy, 2005; Nussey et al., 2013; Vidal et al., 2013), (b) causas (Aguiar et al., 2014; Bai et al., 2015; Hernández \& Gutiérrez 2014; Hill, 2015; McCarty et al., 2015; Montoya et al., 2014; Myers et al., 2010; Vidal et al., 2013; Young, 1999), (c) prejuízos decorrentes do mesmo (Bai et al., 2015; Hill, 2015; Hirvikoski et al., 2015; Hogue et al., 2014; Kądziela-Olech, 2012; McCarty et al., 2015; McCleary \& Ridley, 1999; Murphy, 2005; Vidal et al., 2013) e (d) comorbidades existentes (Hill, 2015; McCarty et al., 2015; Mesquita et al., 2009; Montoya et al., 2011; Montoya et al., 2014).

A psicoeducação do tratamento do TDAH tratou de temas como a medicação (Asherson, 2012; Bai et al., 2015; Coletti et al., 2012; Hill, 2015; Hirvikoski et al., 2015; Mesquita et al., 2009; Vidal et al., 2013) e as estratégias para manejo dos sintomas (Ferrin et al., 2014; Hernández \& Gutiérrez 2014; Hirvikoski et al., 2015; McCarty et al., 2015; McCleary \& Ridley, 1999; Montoya et al., 2014; Myers et al., 2010). Nos casos da psicoeducação de familiares de crianças/adolescentes com TDAH, foram tratadas também as habilidades parentais necessárias para se relacionar com o filho, manejar os sintomas do transtorno (Kądziela-Olech, 2012; Levine \& Anshel, 2011; Long et al., 1993; McCarty et al., 2015; Montoya et al., 2011; Montoya et al., 2014).

Um dos estudos analisados não abordou psicoeducação propriamente dita, mas investigou os conhecimentos de adolescentes (com alto e baixo risco de apresentar TDAH) e genitores sobre o transtorno. Os participantes relataram informações incorretas sobre o tema (como o transtorno ser decorrente de elevado consumo de açúcar ou uso indevido de medicação) embora acreditassem estar familiarizados com o mesmo, o que evidencia a necessidade de ampliar o acesso a informações sobre o TDAH. Ademais, tanto adolescentes quanto os genitores costumam buscar informações sobre o tema na internet, redes 
sociais, e televisão, mas apenas os genitores procuram também informativos escritos e profissionais da saúde (Bussing et al., 2012). Em outro estudo analisado, verificou-se que os genitores valorizam a tentativa do profissional de explicar o transtorno e o uso da medicação. Contudo, a preferência pela quantidade de informações e modalidade de transmissão destas variou entre os participantes: alguns pais preferiram informações detalhadas em forma de panfleto e/ou internet, enquanto outros, informações resumidas transmitidas de forma oral pelo profissional da saúde (Coletti et al., 2012). Esses resultados indicam as fontes que podem ser utilizadas para acessar o público-alvo na ocasião de psicoeducação do TDAH.

Outro estudo avaliou os conhecimentos e crenças de professores de escolas públicas e privadas sobre o TDAH. A maioria dos professores considerou o TDAH um transtorno mental (79,3\%) e o psicólogo como o profissional competente para realizar o diagnóstico e tratamento do mesmo. Menos da metade $(44,1 \%)$ dos professores acredita que o tratamento combinado é a opção ideal, e apenas $14,7 \%$ pensa que a medicação é o componente principal do tratamento. Mesmo assim, reconhecem os efeitos do tratamento no funcionamento social e acadêmico dos alunos com TDAH (Palacios-Cruz et al., 2013). Esses resultados indicam a necessidade de psicoeducar os professores sobre a etiologia do TDAH, bem como opções de tratamento e eficácia. Afinal, os professores convivem com os alunos diariamente e podem auxiliar na identificação e encaminhamento daqueles que podem apresentar o transtorno.

\section{Público-Alvo}

Um dos fatores que determinam o formato da psicoeducação é o público-alvo ao qual se destina. Na amostra analisada, a psicoeducação foi realizada para uma ou mais populações. A maioria dos estudos desenvolveu a psicoeducação destinada a familiares de crianças e adolescentes com TDAH (Anderson \& Guthery, 2015; Bai et al., 2015; Coletti et al., 2012; Ferrin et al., 2014; Hernández \& Gutiérrez 2014; Hill, 2015;
Hogue et al., 2014; Janssen et al., 2014; KądzielaOlech, 2012; Korzeniowsk \& Ison, 2008; Levine \& Anshel, 2011; Long et al., 1993; McCarty et al., 2015; McCleary \& Ridley, 1999; Montoya et al., 2011; Montoya et al., 2014; Myers et al., 2010; Nussey et al., 2013) ou de adultos com TDAH (Hirvikoski et al., 2015). Um estudo foi realizado com crianças com TDAH (Levine \& Anshel, 2011), quatro com adolescentes com TDAH (Asherson, 2012; Bussing et al., 2012; Hogue et al., 2014; Murphy, 2005) e oito com adultos com TDAH (Altin et al., 2016; Asherson, 2012; Hirvikoski et al., 2015; Knouse et al., 2008; Mesquita et al., 2009; Murphy, 2005; Vidal et al., 2013; Young, 1999). Os demais estudos abordaram psicoeducação do TDAH para professores (Aguiar et al., 2014; Hernández \& Gutiérrez 2014; Hill, 2015; Kądziela-Olech, 2012; Korzeniowsk \& Ison, 2008; Montoya et al., 2011; Nussey et al., 2013; Palacios-Cruz et al., 2013) e para profissionais da saúde básica (Hill, 2015).

Verifica-se uma maior preocupação dos pesquisadores em psicoeducar os familiares do que os próprios pacientes com TDAH. Há duas explicações possíveis para esse resultado. A primeira justificativa é que, historicamente, o TDAH era considerado um transtorno exclusivo da infância. Assim, a realização do tratamento do filho com TDAH dependia do engajamento dos genitores, o que justifica a necessidade de psicoeducar os familiares de crianças e adolescentes com o transtorno. As evidências de que o TDAH interfere na autoestima dos pacientes (APA, 2013) demonstraram a necessidade de adaptar a psicoeducação também para crianças, adolescentes e adultos em 2004 (Lukens \& McFarlane, 2004). Contudo, mais de 10 anos depois, a quantidade de relatos da psicoeducação do TDAH para esse público ainda é inferior aos destinados para seus familiares. A segunda explicação possível é que o TDAH em adultos começou a ser estudado apenas nos últimos anos, e foi inserido recentemente no Manual Diagnóstico e Estatístico dos Transtornos Mentais (DSM5; APA, 2013), o que pode justificar o menos número de estudos sobre psicoeducação para essa 
população. Apesar das lacunas identificadas nos relatos de psicoeducação para públicos diversos, recomenda-se psicoeducação da população em geral sobre o TDAH, visto que pode contribuir não só para identificação de casos não tratados, mas também para aumentar atitudes positivas frente às pessoas com esse transtorno (Nussey et al., 2013).

\section{Variáveis Relacionadas à Psicoeducação do TDAH}

A amostra analisada relacionou psicoeducação do TDAH com maior adesão ao tratamento por parte de pacientes e familiares (Bai et al., 2015; Coletti et al., 2012; Hirvikoski et al., 2015; Janssen et al., 2014; McCarty et al., 2015; Montoya et al., 2014; Myers et al., 2010; Young, 1999), menores níveis de estresse (Anderson \& Guthery, 2015; Ferrin et al., 2014; Hirvikoski et al., 2015), maiores níveis de autoestima (Young, 1999), maior conhecimento sobre o transtorno (Aguiar et al., 2014; Bai et al., 2015; Hirvikoski et al., 2015), maiores índices de qualidade de vida (Ferrin et al., 2014; Hirvikoski et al., 2015; Janssen et al., 2014; Vidal et al., 2013), menor presença de sintomas do TDAH e de outras psicopatologias (Ferrin et al., 2014; Janssen et al., 2014; Kądziela-Olech, 2012; Korzeniowsk \& Ison, 2008; Levine \& Anshel, 2011; Long et al., 1993; Mesquita et al., 2009; Myers et al., 2010; Vidal et al., 2013), maior colaboração entre os genitores e a escola, e maiores níveis de desempenho escolar (Hogue et al., 2014).

As variáveis associadas com a psicoeducação do TDAH nos estudos analisados vão ao encontro das variáveis utilizadas para mensurar impacto nas intervenções em psicoeducação de maneira geral. Evidências sobre programas de psicoeducação em geral mostram que a mesma parece estar associada com a redução dos sintomas psicoeducados, a redução de sintomas associados a ansiedade e a depressão (independente do foco da psicoeducação), maior adesão e satisfação com o tratamento, maior conhecimento, autoestima, estratégias de enfrentamento e qualidade de vida (Lukens \& McFarlane, 2004).

\section{Características das Intervenções em Psicoeducação do TDAH}

$\mathrm{Na}$ amostra analisada, 17 estudos apresentaram intervenções psicoeducativas do TDAH para pessoas com diagnóstico ou traços do transtorno. O diagnóstico foi realizado pela triangulação de dados em cinco pesquisas (Bai et al., 2015; Ferrin et al., 2014; Hirvikoski et al., 2015; Levine \& Anshel 2011; Montoya et al., 2014). De fato, o diagnóstico do TDAH é clínico e requer a coleta de informações em diferentes fontes, como escalas e entrevistas semiestruturadas baseadas nos critérios do DSM-5 com o paciente, coleta de informações com os familiares do indivíduo, identificação dos contextos em que os sintomas nucleares se mostram presentes, bem como o comprometimento funcional associado aos mesmos (Mattos et al., 2006). Os demais estudos analisados basearam o diagnóstico apenas em critérios diagnósticos (Hogue et al., 2014; Kądziela-Olech, 2012; Vidal et al., 2013), escalas (McCarty et al., 2015; Mesquita et al., 2009; Vidal et al., 2013), entrevistas clínicas (Janssen et al., 2014; Long et al., 1993; McCleary \& Ridley, 1999; Myers et al., 2010) ou confiança em diagnóstico prévio (Korzeniowsk \& Ison, 2008), o que pode levar a erros de diagnóstico devido aos sintomas do TDAH serem comuns a outros transtornos, como transtornos do humor e da ansiedade (Searight, Burke, \& Rootnek, 2000).

A maioria das intervenções consistia em um conjunto de sessões interativas grupais (Ferrin et al., 2014; Hirvikoski et al., 2015; Hogue et al., 2014; Korzeniowsk \& Ison, 2008; McCarty et al., 2015; McCleary \& Ridley, 1999; Montoya et al., 2014; Vidal et al., 2013), enquanto as demais ocorreram em formato de palestras associadas a manual (Aguiar et al., 2014; Bai et al., 2015), biblioterapia (Anderson \& Guthery, 2015; Long et al., 1993) e encontros individuais com o público-alvo (Levine \& Anshel, 2011; Mesquita et al., 2009). Dois estudos analisados apenas informaram quem recebeu psicoeducação, sem descrever como a mesma foi realizada (Janssen et al., 2014; Myers et al., 2010). Outra pesquisa relata o acompanhamento de uma intervenção 
psicoeducativa mensal para pais e professores de crianças com TDAH após um e 10 anos, mas não descreveu como a psicoeducação foi realizazda (duração, individual ou grupal, local, equipe; Kądziela-Olech, 2012).

Em todas as pesquisas, foram utilizados questionários e escalas para avaliação das variáveis descritas na categoria anterior em, pelo menos, dois tempos (pré e pós intervenção), com exceção de três estudos que realizaram avaliação qualitativa (Hogue et al., 2014; Korzeniowsk \& Ison, 2008; Levine \& Anshel, 2011). Constata-se que medidas de processo, tais como presença nas sessões, taxa de evasão, treinamento dos facilitadores, raramente foram avaliadas. $\mathrm{O}$ estudo de Aguiar et al. (2014) descreveu o treinamento oferecido à equipe profissional que conduziu a intervenção e o de Myers et al. (2010) avaliou a presença dos pais nas sessões de intervenção.

Tabela 2

Características das Intervenções em Psicoeducação do TDAH

\begin{tabular}{|c|c|c|}
\hline $\begin{array}{l}\text { Autores } \\
\text { (ano) }\end{array}$ & Diagnóstico de TDAH & Modelo de intervenção \\
\hline
\end{tabular}

\begin{tabular}{cc}
\hline Aguiar et al. (2014) & $\begin{array}{c}\text { Não houve } \\
\text { (professores do } \\
\text { Ensino Fundamental) }\end{array}$
\end{tabular}

Anderson \& Guthery (2015)

Bai et al. (2015)

Ferrin et al. (2014) Baseado no DSMIV, confirmado por entrevista clínica com psiquiatra, entrevista estruturada com KSADS-PL
Crianças com TDAH ou traços do TDAH

Uma sessão de seis horas: vinhetas clínicas para discutir mitos sobre o TDAH, sintomas, etiologia e estratégias para lidar com os transtornos. Manual com resumo dos conteúdos trabalhados.

Biblioterapia para pais (Everyday Blessings: The Inner Work of Mindful Parenting)

Baseado no DSM-IV, no Barkley's Clinical Diagnostic Interview Scale, e realizado por psiquiatra
Duas sessões de 40 minutos com até 10 pais para fornecer informações sobre efeitos colaterais e responder dúvidas.

Palestra e manual para pais sobre: 1- TDAH: sintomas, causas, prejuízos ao longo da vida, 2- Eficácia de tratamento farmacológico e não farmacológico, efeitos colaterais da medicação, 3- Barreiras para aderência ao tratamento e estratégias de enfrentamento, 4- Habilidades parentais

O manual contém formulários para controlar doses da medicação e efeitos colaterais.

Comunidade online com materiais sobre saúde, comunicação com outros pais e com profissional responsável pelas intervenções.

12 sessões semanais de 90 minutos para grupos de até 10 familiares.

Sessões 1-9: TDAH, Sessões 10-12: estratégias comportamentais para manejo dos sintomas do TDAH e redução do comportamento desafiante.

Hand-outs no final de cada sessão.
Questionário para avaliar conhecimentos sobre o TDAH (sintomas, etiologia, estratégias para lidar com o transtorno e tratamento)

PSI-4-SF, Evaluation of the Mindful

Parenting Education

Study, satisfação com intervenção.

Questionário sobre TDAH e medicação, perguntas sobre aderência à medicação, perguntas sobre modelo

TPB, ADHD Rating

Scale-IV, satisfação com programa

K-SADS-PL, Conners' Parent Rating Scale

Revised 27-items version, Strengths and Difficulties Questionnaire, Clinical Global Impression Scale,

PSI-SF, EuroQoL Five Dimension Questionnaire, PedsQLTM Family Impact Module 


\begin{tabular}{|c|c|c|c|}
\hline $\begin{array}{l}\text { Autores } \\
\text { (ano) }\end{array}$ & Diagnóstico de TDAH & Modelo de intervenção & $\begin{array}{l}\text { Instrumentos } \\
\text { para avaliação }\end{array}$ \\
\hline
\end{tabular}

Hirvikoski et al. Baseado em entrevista (2015)

Hogue et al. (2014) Critérios diagnósticos para TDAH

Janssen et al.

(2014)

Kądziela-Olech (2012) clínica de acordo com DSM-IV-TR, WURS, ASRS, contato com familiares
Entrevista clínica

Critérios diagnósticos do CID-10 minutos com 30 minutos de intervalo)

em grupo (20-30 pessoas com TDAH e outros significativos)

1-TDAH na vida adulta, 2-Tratamento farmacológico

e psicológico, 3- Fatores associados ao estilo de vida, 4- Estratégias para lidar com dificuldades decorrentes dos sintomas, 5 Como é viver com TDAH, 6- TDAH nos relacionamentos, 7- TDAH

no trabalho, 8- Serviços de suporte

Método: material audiovisual e discussões em grupo.

\section{CASH-AA}

1- Psicoeducação do TDAH (prevalência, sintomas, impacto no desenvolvimento, neurobiologia para maior aceitação da família, menor atribuição moral e expectativas realistas de mudança, benefícios da medicação, funções executivas, desempenho acadêmico),

2- Técnicas motivacionais para engajamento da família, 3- Treinamento para planejamento dos temas de casa e habilidades de organização

Uso do ADHD Style Index e de checklists (Problem Scorecards) para promover psicoeducação sobre sintomas do TDAH e déficits nas funções executivas. Não há informações
Visitas mensais aos pais que abordaram:

1- Diagnóstico, 2- Dificuldades que a criança pode encontrar na educação, com familiares e pares,

3- Regulação do estilo de vida da criança no que se refere à educação e hábitos,

4- Controle de estímulos e da ansiedade, 5- Ajuste das expectativas parentais em relação à criança

Transmissão de informações aos professores sobre a natureza do TDAH e como lidar com as crianças na escola.
ADHD 20 questions,

QAFM, Rosenberg's

Self-Esteem Scale,

Adult Attention Deficit/

Hyperactivity Disorder Quality-of-Life

(AAQoL) Scale, Burden Assessment Scale

Não houve.

ADHD Rating Scale, Health of the Nation Outcome Scale, Kidscreen-10, Morisky Adherence Scale

Diagnostic Interview Schedule for Children 


\begin{tabular}{|c|c|c|c|}
\hline $\begin{array}{l}\text { Autores } \\
\text { (ano) }\end{array}$ & Diagnóstico de TDAH & Modelo de intervenção & $\begin{array}{c}\text { Instrumentos } \\
\text { para avaliação }\end{array}$ \\
\hline
\end{tabular}

Korzeniowsk \& Ison (2008)
Levine \& Anshel (2011)

Long et al. (1993)
Observações da criança, entrevista com mãe, professor e criança, National Initiative for Children's Healthcare Quality (NICHQ, baseada no DSM-IV), Teacher Rating Scale of the BASC-II

\section{Entrevista clínica com pediatra}

McCarty et al. (2015)

Checklist e Diagnóstico prévio
de TDAH
Oito sessões semanais para pais. Tópicos:

1- Informações sobre o TDAH,

2- Fatores que interferem no comportamento de crianças com TDAH, 3- Dificuldades escolares e estratégias de organização e atenção, 4- Economia de fichas, 5- Uso de agenda escolar, 6- Imposição de limites, 7- Identificação de recursos,8- Resolução de problemas Oito sessões semanais para professores. Tópicos:

1- Informações sobre o TDAH, 2- Ajuste entre criança com TDAH e contexto educacional, 3- Assistência nas habilidades de organização e estudo, 4- Atenção e motivação de crianças com TDAH, 5- Estratégias para abordar hiperatividade e impulsividade em sala de aula, 6- Manejo de comportamentos e relações interpessoais, 7- Otimização de estratégias, 8- Problemas futuros

Tópicos abordados: 1- Intervenções cognitivo-comportamentais (comportamento, escola, casa), 2- Treinamento de pais e professores, 3- Literatura sobre TDAH, informações online e lista de grupos de apoio para a mãe, 4- Psicoeducação com a criança sobre TDAH, TCC e estratégias de enfrentamento

Biblioterapia: técnicas de manejo do comportamento opositor da criança (recompensar, ignorar, dar instruções, time-out).

Seis sessões semanais. Tópicos:

Computerized

Diagnostic Interview Schedule for Children
Observações da criança em sala de aula, entrevista com mãe e professora.
Conners Parent Rating Scale-Hyperactivity Index (CPRS-HI),

Eyberg Child Behavior Inventory (ECBI), Behavior Rating Profile-Teacher Rating Scale (BRPT),

Knowledge of Behavioral Principles as Applied to Children (KBPAC)

Child Behavior Checklist, Computerized Diagnostic Interview Schedule for Children, IOWA Conners' Rating Scale, Global Assessment of Improvement scale
1- Entendendo o TDAH e seu filho, 2- Escola e crianças com TDAH, 3- Habilidades de enaltecer e ignorar, 4- Fornecendo instruções claras e mantendo-as, 5- Time-out e outras consequências ou sistema de pontos por comportamento, 6- Revisão 


\begin{tabular}{|c|c|c|c|}
\hline $\begin{array}{l}\text { Autores } \\
\text { (ano) }\end{array}$ & Diagnóstico de TDAH & Modelo de intervenção & $\begin{array}{c}\text { Instrumentos } \\
\text { para avaliação }\end{array}$ \\
\hline
\end{tabular}

McCleary \&

Ridley (1999)

Mesquita et al. (2009)

Montoya et al.

(2014)

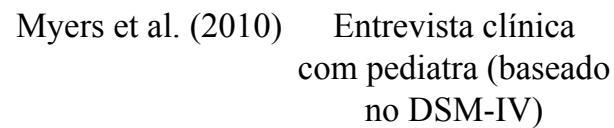

Vidal et al. (2013) Critérios do DSM-IV, ADHD-RS e CGI-S

Critérios

do DSM-IV

e ASRS

Critérios do ADHD-RS-IV Parent: Inv, Clinical Global Impression-ADHD Severity

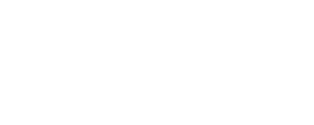

DSM-IV-TR,
10 sessões semanais de 120 minutos em grupo de 8-24 pais

Tópicos: resolução de problemas, conhecendo seu filho de novo, ignorar deliberadamente, planejamento de contingências, time-out, relação com irmãos, problemas escolares, relações com pares.

20 sessões semanais de 50 minutos com foco na redução dos sintomas depressivos e do TDAH.

Técnicas: psicoeducação, solução de problemas, questionamento socrático, reestruturação cognitiva, uso de agenda, lembretes e alarmes, estabelecimento de prioridades, manejo de tempo e organização

Cinco sessões semanais de 90 minutos em grupos de até seis pais. Tópicos:

1- Psicoeducação sobre o programa e o TDAH, 2- Técnicas de reforçamento, 3- Técnicas para lidar com comportamentos inadequados, habilidades sociais, emocionais e parentais,

4- Intervenções para problemas de atenção, 5- Recaptulação das sessões anteriores

Apenas descreve que os pais foram psicoeducados sobre etiologia e manejo do TDAH

12 sessões semanais de duas horas com 7-8 participantes. Tópicos: 1- Mitos e realidades no TDAH,

2- Diagnóstico e características do TDAH, 3- Causas e tratamentos do TDAH,

4- Sessão com um membro da família, 5- Sintomas positivos e negativos, 6- Relaxamento, 7- Planificação no TDAH, 8- Resolução de problemas no TDAH, 9- Atenção no TDAH, 10- Modelo Cognitivo do TDAH
ADHD-RS-IV Parent: Inv, CGI-ADHD-S, WFIRS-P, Treatment satisfaction questionnaire.
Questionário

Conflict Behavior Questionnaire (CBQ);

Issues Checklist $\mathrm{e}$ questionário qualitativo específico ao final do programa.

BDI, BAI e ASRS
Vanderbilt ADHD Rating Scales-Parent (VADPRS) and Teacher (VADTRS) versions, entrevistas, número de sessões em que participaram

ADHD-RS, CAARS-S, CGI-S, BDI, STAI-S, Quality of Life Enjoyment and Satisfaction Questionnaire (QLESQ)
Os pacientes tendem a apreender apenas $50 \%$ das informações relevantes transmitidas pelo profissional (Swaminath, 2009), o que sugere a necessidade de avaliar as informações absorvidas pelos pacientes. Apenas três estudos verificaram se as informações fornecidas na psicoeducação do TDAH foram, de fato, compreendidas por pacientes, familiares e professores. Bai et al. (2015) utilizaram um questionário composto por 16 itens para avaliar o conheci- 
mento dos genitores de crianças com TDAH. Cinco itens referiam-se ao TDAH (sintomas nucleares, causas, prejuízos, se é crônico) e 11, à medicação (tratamento combinado, diminuição dos sintomas do TDAH, possibilidade de dependência, efeitos colaterais). Após a intervenção, os participantes do grupo experimental demonstraram conhecimento significativamente maior que o grupo controle e maior tendência a aderir ao tratamento (Bai et al., 2015). Hirvikoski et al. (2015) também avaliaram o conhecimento dos participantes por meio de um questionário. O ADHD 20 Questions é composto por 20 itens sobre início do TDAH, possibilidade de contágio, sintomas, possíveis prejuízos, comorbidades etc. (Bramham et al., 2009). A intervenção realizada resultou em maior conhecimento sobre o transtorno, bem-estar psicológico e qualidade do relacionamento entre adultos com TDAH e familiares (Hirvikoski et al., 2015). Aguiar et al. (2014) avaliaram o conhecimento de professores do Ensino Fundamental sobre o TDAH por meio de instrumento construídos pelos próprios pesquisadores baseado em itens da literatura. Como resultado, verificou-se uma redução no nível de crenças incorretas sobre o TDAH (Aguiar et al., 2014). O uso de instrumentos como esses pode auxiliar na identificação de informações distorcidas a serem abordadas durante a psicoeducação, bem como servir como forma de avaliar o quanto a psicoeducação foi efetiva para o público-alvo.

\section{Considerações Finais}

Este estudo apresenta as características da psicoeducação do TDAH a partir de publicações nacionais e internacionais. Após análise dos 29 estudos que satisfaziam os critérios de inclusão, constatou-se que nem todas as publicações apresentam claramente o conceito de psicoeducação utilizado, o que pode confundir os leitores dada a natureza teoricamente diversificada do constructo. A psicoeducação tem sido destinada, na maioria dos estudos, a familiares de pessoas com TDAH. Em geral, a psicoeducação deste transtorno parece contribuir para maior conhecimento sobre ele, maior adesão ao tratamento, menor intensidade dos sintomas, bem como maior qua- lidade de vida. Contudo, poucos estudos avaliaram o quanto as informações fornecidas na psicoeducação foram, de fato, apreendidas pelo público-alvo.

Destacam-se algumas limitações que podem interferir na interpretação dos resultados apresentados. A revisão de literatura foi realizada com a combinação de apenas cinco descritores e em apenas dois idiomas. É possível que outros artigos sobre o mesmo tema não tenham sido identificados e analisados. Logo, os resultados apresentados referem-se apenas à amostra de artigos selecionados para análise neste estudo.

A maior contribuição desta revisão sistemática foi oferecer um panorama da produção nacional e internacional sobre psicoeducação do TDAH a partir da busca em oito bases de dados, o que permitiu identificar aspectos que necessitam de maior atenção dos pesquisadores. Constatou-se ausência de relato de psicoeducação do transtorno para professores de nível superior e profissionais da saúde, o que sugere a necessidade de desenvolver adaptações da psicoeducação sobre o próprio transtorno e tratamento para esse público. Verificou-se também que já se sabe o conteúdo que deve ser transmitido em psicoeducação do TDAH, embora os profissionais pudessem investir em outros meios (internet, redes sociais, manuais) para atingir adolescentes e genitores, por exemplo.

\section{Referências}

Aguiar, A. P., Kieling, R. R., Costa, A. C., Chardosim, N., Dorneles, B. V., Almeida, M. R., ...Rohde, L. A. (2014). Increasing teachers' knowledge about ADHD and learning disorders: An investigation on the role of a psychoeducational intervention. Journal of Attention Disorders, 18(8), 691-698. doi:10.1177/1087054712453171

Altin, M., Altin, G. E., \& Semerci, B. (2016). An online survey of Turkish psychiatrists' attitudes about and experiences of adult attention deficit hyperactivity disorder in clinical practice. $\mathrm{Neu}$ ropsychiatric Disease and Treatment, 12, 2455 2461. doi:10.2147/NDT.S110720

Anderson, S. B., \& Guthery, A. M. (2015). Mindfulness-based psychoeducation for parents of children with Attention-Deficit/Hyperactivity 
Disorder: An applied clinical project. Journal of Child and Adolescent Psychiatric Nursing, 28(1), 43-49. doi:10.1111/jcap.12103

American Psychiatric Association. (2013). Diagnostic and statistical manual of mental disorders ( $5^{\text {th }}$ ed.). Arlington, VA: Author.

Asherson, P. (2012). ADHD across the lifespan. Medicine, 40(11), 623-627. doi:http://dx.doi. org/10.1016/j.mpmed.2012.08.007

Bai, G. N., Wang, Y. F., Yang, L., \& Niu, W. Y. (2015). Effectiveness of a focused, brief psychoeducation program for parents of ADHD children: improvement of medication adherence and symptoms. Neuropsychiatric Disease and Treatment, 11, 2721-2735. doi:10.2147/NDT.S88625

Barkley, R. A., Fischer, M., Smallish, L., \& Fletcher, K. (2002). The persistence of attention-deficit/ hyperactivity disorder into young adulthood as a function of reporting source and definition of disorder. Journal of Abnormal Psychology, 111(2), 279-89. doi:http://dx.doi.org/10.1037/0021843X.111.2.279

Bäuml, J., Froböse, T., Kraemer, S., Rentrop, M., \& Pitschel-Walz, G. (2006). Psychoeducation: A basic psychotherapeutic intervention for patients with schizophrenia and their families. Schizophrenia Bulletin, 32(1), 51-59. doi:10.1093/ schbul/sbl017

Bégin, J. Y., Bluteau, J., Arseneault, C., \& Pronovost, J. (2012). Psychoeducation in Quebec: Past to Present. Ricerche di Pedagogia e Didattica. Journal of Theories and Research in Education, 7(1), 1-16. doi:10.6092/issn.1970-2221/2681

Bonsack, C., Rexhaj, S., \& Favrod, J. (2015). Psychoéducation: définition, historique, intérêt et limites. Annales Médico-psychologiques, 173(1), 79-84. doi:10.1016/j.amp.2014.12.001

Bramham, J., Young, S., Bickerdike, A., Spain, D., McCartan, D., \& Xenitidis, K. (2009). Evaluation of group cognitive behavioral therapy for adults with ADHD. Journal of Attention Disorders, 12(5), 434-441. doi:10.1177/1087054708314596

Burlingame, G., Ridge, N., Matasuno, J., Hwang, A., \& Ernshaw, D. (2006). Educational needs of inpatients with severe and persistent mental illnesses. Journal of Psychosocial Nursing, 44(1), $39-43$.

Bussing, R., Zima, B. T., Mason, D. M., Meyer, J. M., White, K., \& Garvan, C. W. (2012). ADHD knowledge, perceptions, and information sources: Perspectives from a community sample of adolescents and their parents. Journal of Adolescent Health, 51(6), 593-600. doi:10.1016/j. jadohealth.2012.03.004

Charach, A., \& Fernandez, R. (2013). Enhancing ADHD medication adherence: Challenges and opportunities. Current Psychiatry Reports, 15(7), 1-8. doi:10.1007/s11920-013-0371-6

Coletti, D. J., Pappadopulos, E., Katsiotas, N. J., Berest, A., Jensen, P. S., \& Kafantaris, V. (2012). Parent perspectives on the decision to initiate medication treatment of attention-deficit/hyperactivity disorder. Journal of Child and Adolescent Psychopharmacology, 22(3), 226-237. doi:10.1089/cap.2011.0090

Conners, C. K. (2009). Diagnóstico e avaliação do TDAH. In C. K. Conners, Transtorno de déficit de Atenção/hiperatividade: As mais recentes estratégias de avaliação e tratamento. Porto Alegre, RS: Artmed.

Ferrin, M., Moreno-Granados, J. M., Salcedo-Marin, M. D., Ruiz-Veguilla, M., Perez-Ayala, V., \& Taylor, E. (2014). Evaluation of a psychoeducation programme for parents of children and adolescents with ADHD: Immediate and long-term effects using a blind randomized controlled trial. European Child \& Adolescent Psychiatry, 23(8), 637-647. doi:10.1007/s00787-013-0494-7

Ferrin, M., Perez-Ayala, V., El-Abd, S., Lax-Pericall, T., Jacobs, B., Bilbow, A., \& Taylor, E. (2016). A randomized controlled trial evaluating the efficacy of a psychoeducation program for families of children and adolescents with ADHD in the United Kingdom: Results after a 6-month follow-up. Journal of Attention Disorders, 1-12. doi:10.1177/1087054715626509

Gil, A. C. (2006). Métodos e técnicas de pesquisa social. São Paulo, SP: Atlas.

Hernández, P. R., \& Gutiérrez, I. C. (2014). Plan de tratamiento multimodal del TDAH. Tratamiento psicoeducativo. Pediatría Integral, 18(9), 624633.

Hill, P. (2015). Attention-deficit hyperactivity disorder in children and adolescents: Assessment and treatment. BJPsych Advances, 21(1), 23-30. doi:10.1192/apt.bp.114.013953

Hirvikoski, T., Waaler, E., Lindström, T., Bölte, S., \& Jokinen, J. (2015). Cognitive behavior therapybased psychoeducational groups for adults with 
ADHD and their significant others (PEGASUS): An open clinical feasibility trial. ADHD Attention Deficit and Hyperactivity Disorders, 7(1), 89-99. doi:10.1007/s12402-014-0141-2

Hogue, A., Bobek, M., \& Evans, S. W. (2014). Changing Academic Support in the home for adolescents with Attention-Deficit/Hyperactivity Disorder: A family-based clinical protocol for improving school performance. Cognitive and Behavioral Practice, 23, 14-30. doi:10.1016/j. cbpra.2014.07.002

Janssen, M., Wensing, M., van der Gaag, R. J., Cornelissen, I., van Deurzen, P., \& Buitelaar, J. (2014). Improving patient care for attention deficit hyperactivity disorder in children by organizational redesign (Tornado program) and enhanced collaboration between psychiatry and general practice: A controlled before and after study. Implementation Science, 9(1), 155-162. doi:10.1186/s13012-014-0155-3

Kądziela-Olech, H. (2012). Attention-deficit/hyperactivity disorder in children - The role of psychoeducation in longitudinal study. Psychiatria i Psychologia Kliniczna, 12(3), 149-156.

Knapp, P. (2004). Terapia Cognitivo-Comportamental na Prática Psiquiátrica. Porto Alegre, RS: Artmed.

Knouse, L. E., Cooper-Vince, C., Sprich, S., \& Safren, S. A. (2008). Recent developments in the psychosocial treatment of adult ADHD. Expert Review of Neurotherapeutics, 8(10), 1537-1548. doi:10.1586/14737175.8.10.1537

Korzeniowsk, C., \& Ison, M. (2008). Estrategias psicoeducativas para padres y docentes de niños con TDAH. Revista Argentina de Clínica Psicológica, 18(1), 65-71.

Lara, C., Fayyad, J., De Graaf, R., Kessler, R. C., Aguilar-Gaxiola, S., Angermeyer, M., ...Karam, E. G. (2009). Childhood predictors of adult attention-deficit/hyperactivity disorder: Results from the World Health Organization World Mental Health Survey Initiative. Biological Psychiatry, 65(1), 46-54. doi:10.1016/j.biopsych.2008.10.005

Levine, E. S., \& Anshel, D. J. (2011). "Nothing works!" A case study using cognitive-behavioral interventions to engage parents, educators, and children in the management of attentiondeficit/hyperactivity disorder. Psychology in the Schools, 48(3), 297-306. doi:10.1002/pits.20554
Long, N., Rickert, V. I., \& Ashcraft, E. W. (1993). Bibliotherapy as an adjunct to stimulant medication in the treatment of attention-deficit hyperactivity disorder. Journal of Pediatric Health Care, 7(2), 82-88. doi:10.1016/08915245(93)90078-V

Lukens, E. P., \& McFarlane, W. R. (2004). Psychoeducation as evidence-based practice: Considerations for practice, research, and policy. Brief Treatment and Crisis Intervention, 4(3), 205. doi:10.1093/brief-treatment/mhh019

Mattos, P., Palmini, A., Salgado, C. A., Segenreich, D., Grevet, E., Oliveira, I. R., ...Lima, P. P. (2006). Painel brasileiro de especialistas sobre o diagnóstico do Transtorno do Déficit de Atenção/Hiperatividade (TDAH) em adultos. Revista de Psiquiatria, 28(1), 50-60.

McCarthy, S. (2014). Pharmacological interventions for ADHD: How do adolescent and adult patient beliefs and attitudes impact treatment adherence? Patient Preference \& Adherence, 8, 13171327.

McCarty, C. A., Vander Stoep, A., Violette, H., \& Myers, K. (2015). Interventions developed for psychiatric and behavioral treatment in the Children's ADHD Telemental Health Treatment Study. Journal of Child and Family Studies, 24(6), 1735-1743. doi:10.1007/s10826-014-9977-5

McCleary, L., \& Ridley, T. (1999). Parenting adolescents with ADHD: Evaluation of a psychoeducation group. Patient Education and Counseling, 38(1), 3-10. doi:10.1016/S07383991(98)00110-4

Menezes, S. L., \& Souza, M. C. B. (2012). Implicações de um grupo de psicoeducação no cotidiano de portadores de transtorno afetivo bipolar. Revista da Escola de Enfermagem da USP, 46(1), 124131. doi:10.1590/S0080-62342012000100017

Mesquita, C. M. D., Porto, P. R., Rangé, B. P., \& Ventura, P. R. (2009). Terapia cognitivo-comportamental e o TDAH subtipo desatento: Uma área inexplorada. Revista Brasileira de Terapias Cognitivas, 5(1), 35-45. doi:10.5935/18085687.20090004

Mongia, M., \& Hechtman, L. (2016). AttentionDeficit Hyperactivity Disorder across the lifespan: Review of literature on Cognitive Behavior Therapy. Current Developmental Disorders Reports, 3(1), 7-14. doi:10.1007/s40474-0160072-z 
Montoya, A., Colom, F., \& Ferrin, M. (2011). Is psychoeducation for parents and teachers of children and adolescents with ADHD efficacious? A systematic literature review. European Psychiatry, 26(3), 166-175. doi:10.1016/j.eurpsy.2010.10.005

Montoya, A., Hervás, A., Fuentes, J., Cardo, E., Polavieja, P., Quintero, J., \& Tannock, R. (2014). Cluster-randomized, controlled 12-month trial to evaluate the effect of a parental psychoeducation program on medication persistence in children with attention-deficit/hyperactivity disorder. Neuropsychiatric Disease and Treatment, 10, 1081-1092. doi:10.2147/NDT.S62487

Murphy, K. (2005). Psychosocial treatments for ADHD in teens and adults: A practice-friendly review. Journal of Clinical Psychology, 61(5), 607-619. doi:10.1002/jclp.20123

Myers, K., Vander Stoep, A., Thompson, K., Zhou, C., \& Unützer, J. (2010). Collaborative care for the treatment of Hispanic children diagnosed with attention-deficit hyperactivity disorder. General Hospital Psychiatry, 32(6), 612-614. doi:10.1016/j.genhosppsych.2010.08.004

Nussey, C., Pistrang, N., \& Murphy, T. (2013). How does psychoeducation help? A review of the effects of providing information about Tourette syndrome and attention-deficit/hyperactivity disorder. Child: Care, Health and Development, 39(5), 617-627. doi:10.1111/cch.12039

Palacios-Cruz, L., de la Peña Olvera, F., Figueroa, G. V., Caballero, A. A., de la Rosa Muñoz, L., Pedroza, ...Flores, R. E. U. (2013). Knowledge and beliefs about attention deficit hyperactivity disorder in teachers from three Latin-American countries. Salud Mental, 36, 257-262.
Searight, H. R., Burke, J. M., \& Rootnek, K. F. (2000). Adult ADHD: Evaluation and treatment in family medicine. American Family Physician, 62(9), 2077-2086.

Sprich, S. E., Safren, S. A., Finkelstein, D., Remmert, J. E., \& Hammerness, P. (2016). A randomized controlled trial of cognitive behavioral therapy for ADHD in medication-treated adolescents. Journal of Child Psychology and Psychiatry. doi:10.1111/jcpp.12549

Swadi, H., Bobier, C., Price, L., \& Craig, B. (2010). Lessons from an audit of psychoeducation at an older adolescent inpatient unit. Australasian Psychiatry, 18(1), 53-58. doi:10.1080/10398560902721580

Swaminath, G. (2009). Psychoeducation. Indian journal of psychiatry, 51(3), 171-172. doi:10.4103/0019-5545.55082

Vidal, R., Bosch, R., Nogueira, M., Gómez-Barros, N., Valero, S., Palomar, G., ...Ramos-Quiroga, J. A. (2013). Psychoeducation for adults with attention deficit hyperactivity disorder vs. cognitive behavioral group therapy: A randomized controlled pilot study. The Journal of Nervous and Mental Disease, 201(10), 894-900. doi:10.1097/NMD.0b013e3182a5c2c5

Young, S. (1999). Psychological therapy for adults with attention deficit hyperactivity disorder. Counselling Psychology Quarterly, 12(2), 183190. doi:10.1080/09515079908254088

Recebido: $23 / 09 / 2016$

$1^{a}$ revisão: $23 / 02 / 2017$

Aceite final: 28/02/2017

(C) $\mathrm{O}(\mathrm{s})$ autor(es), 2018. Acesso aberto. Este artigo está distribuído nos termos da Licença Internacional Creative Commons Atribuição 4.0 (http://creativecommons.org/licenses/by/4.0/), que permite o uso, distribuição e reprodução sem restrições em qualquer meio, desde que você dê crédito apropriado ao(s) autor(es) original(ais) e à fonte, fornecer um link para a licença Creative Commons e indicar se as alterações foram feitas. 